\title{
Sports de nature, développement durable et controverse environnementale
}

\author{
Jean-Pierre Mounet \\ Sociologue et écologue, Université Joseph Fourier, Laboratoire SENS, BP 53, 38041 Grenoble cedex 9, France
}

\author{
Mots-clés : \\ sociologie; \\ écologie ; \\ développement \\ durable ; \\ sports de nature
}

\section{Keywords:}

sociology; ecology; sustainable development; outdoor sports

\begin{abstract}
Résumé - L'article analyse une recherche-action, dont l'objectif était d'aider les acteurs départementaux à réaliser un Plan départemental des espaces, sites et itinéraires relatifs aux sports de nature, en tenant compte de l'environnement dans le cadre d'un développement durable. Après avoir rapidement présenté ce que sont les sports de nature, il s'appuie sur un examen critique bibliographique pour identifier l'impact environnemental de ces activités. Puis il expose les conditions de la construction d'un instrument d'évaluation environnementale et les jeux d'acteurs qu'il a suscités. La conclusion souligne la difficulté à établir un processus de démocratie participative.
\end{abstract}

\begin{abstract}
Outdoor sports, sustainable development and environmental controversies. This paper aims to highlight the links between outdoor sports, sustainable development and the environment. The action-research work the author analyzed was designed to help local stakeholders manage their interactions and take the environment into account in developing and institutionalizing outdoor sports under a Scheme for spaces, sites and paths relative to outdoor sports.

After briefly presenting the situation of outdoor sports, the article examines critically the literature on the environmental impact of these activities. It then analyzes the conditions in which a tool for environmental evaluation was evolved and the stakeholder interactions it induced. To conclude we show the difficulty that arise in establishing a process of participative democracy.
\end{abstract}

L'engouement des trois dernières décennies pour les sports de nature a conduit le législateur à tenter de les gérer par un Plan départemental des espaces, sites et itinéraires relatifs aux sports de nature (PDESI), dans le cadre d'une commission délibérative réunie sous l'égide du président du conseil général ${ }^{1}$. Une recherche-action a été commandée par le conseil général de l'Ardèche pour la mise au point d'une «méthode de prise en compte du patrimoine naturel ardéchois dans le cadre de la rédaction du PDESI», étayée par « une analyse bibliographique critique de l'impact environnemental de ces activités ${ }^{2} »$.

\footnotetext{
Auteur correspondant : Jean-Pierre.Mounet@ujf-grenoble.fr

${ }^{1}$ Plan départemental (et Commission) des espaces, sites et itinéraires relatifs aux sports de nature (PDESI, CDESI) prévus par la loi sur le sport de juillet 2000.

2 Cette recherche-action, dont nous avons assuré la coordination scientifique, a été menée en 2004, sur la demande du conseil général de l'Ardèche, en collaboration avec deux associations : la FRAPNA Ardèche et Cohérence pour un développement durable. C'est sur ce travail que s'appuie cet article.
}

En s'appuyant sur cet exemple d'institutionnalisation locale des sites sportifs de nature, qui s'inscrit explicitement dans l'idéologie du développement durable, cet article analyse les conditions d'un arbitrage entre la préservation du patrimoine naturel et des activités se réclamant pourtant de la «nature». Quelles sont les données environnementales mobilisables et mobilisées? Comment et par qui le sont-elles? Quels sont les jeux d'acteurs dans cette perspective?

\section{Les sports de nature}

Les «sports de nature » ont longtemps été pratiqués par une «élite » recherchant notamment une distinction sociale (Bourdieu, 1979) dans la confrontation avec la nature. Pour de nombreux auteurs, ces sports se caractérisaient par un jeu avec l'environnement reflétant les compétences intellectuelles des pratiquants de ces «activités physiques de pleine nature » (Pociello, 1981). Le rejet de la chasse, de la pêche et du sport motorisé relevait autant d'une ségrégation sociale que du rapport réel 
à la nature. Celle-ci est plutôt utilisée comme un «terrain de jeu » et, dès 1980, Jacob et Schreyer soulignaient la différence existant entre des pratiques de loisir privilégiant des rapports différents à l'environnement, perception globale et mouvement pour le sport étant opposés à connaissance approfondie et observation pour des activités orientées vers la faune. L'éthique sportive, fondamentalement anthropocentrée ${ }^{3}$, maintes fois citée en réponse aux éthiques environnementales (Larrère, 1997), relève plutôt de conceptions normatives visant à stabiliser une certaine forme de pratique sportive : une «élite » impose sa conception de l'activité en dictant aux autres pratiquants le comportement acceptable pour être agréé en son sein (Jacob et Schreyer, 1980 ; Mounet, 2000).

Puis, la divulgation et la démocratisation de ces activités ont changé la donne en créant brutalement des flux importants qui ont suscité conflits d'usage et mise en cause de leur impact environnemental. De nouveaux acteurs pertinents, aux intérêts parfois divergents, sont alors apparus : les fédérations défendent le sport, les prestataires ${ }^{4}$ leur ressource professionnelle, les collectivités territoriales l'attractivité de leur territoire, les pratiquants indépendants le droit à leur loisir favori et tous, leur passion commune... Des contextes d'action locaux se sont donc structurés en gagnant en complexité.

\section{L'impact environnemental des sports de nature}

Caractériser l'impact des sports de nature revient à considérer qu'ils provoquent des changements significatifs, généralement considérés comme négatifs, sur le milieu naturel. Cette première approche appelle cependant certaines précisions.

Les études d'impact légales concernent un projet localisé, alors que la détermination de l'impact environnemental d'une activité mêle de façon quasi inextricable des acteurs relevant de logiques très différentes et revendique souvent une validité bien plus large.

La nature même de l'impact environnemental pose la question de l'état de référence de l'environnement; or, celui-ci ne peut être défini de manière univoque ni en termes d'équilibre biologique (Lecomte, 2002) ni en termes de paysage «légitime » (Friedberg et al. 2000; Larrère et Larrère, 1997, Lepart et al., 2000).

L'impact pose la question du changement acceptable pour les espèces et les espaces concernés (Stankey et al., 1985) : en connaître les limites nécessiterait de caractériser la «capacité de charge » du site, seuil critique de fréquentation. Mais, si l'on exclut les cas d'impact évident sur

\footnotetext{
${ }^{3}$ Cette affirmation est bien sûr à nuancer pour certaines catégories d'acteurs ou pour certains individus.

${ }^{4}$ Diplômés d'un Brevet d'État d'éducateur sportif ou entreprises de l'offre touristique.
}

milieux sensibles (dunes, zones humides, etc.), les bases du calcul restent hasardeuses et Deprest (1997) souligne l'impossibilité de sa mesure.

Enfin, se pose le problème de la valeur, scientifique ou réglementaire, des entités écologiques concernées, qui renvoie au questionnement de la dichotomie entre nature emblématique/protégée et nature ordinaire (Lecomte, 2002; Mougenot, 2002), ainsi qu'à la disponibilité des données naturalistes (Balland et al., 2003) : malgré un effort et une volonté des acteurs concernés, elles ne sont pas toutes directement accessibles et sont, en outre, encore très incomplètes.

Les sports de nature entraînent parfois des impacts aisément mesurables du fait d'aménagements physiques : l'exemple le plus évident est celui des pistes de ski, qui ont fait l'objet de nombreuses études bien étayées (Cernuska, 1986). Mais il est beaucoup plus difficile de comprendre l'impact de l'activité elle-même.

Une revue bibliographique de 326 documents sert de fondement à cette partie de la réflexion ${ }^{5}$. L'analyse de l'impact d'un sport de nature (Mounet et al., 2000) se réalise en un certain nombre d'étapes qui peuvent servir de grille de lecture pour le dépouillement de la littérature. L'analyse croise d'abord l'activité concernée et le milieu d'accueil. Or, les documents étudiés présentent comme défaut principal de substituer des stéréotypes à l'analyse de l'activité, ce qui limite considérablement leur validité. Winckel (1999, p. 62) offre un exemple de ces constructions de sens commun en affirmant que «les VTT ne respectent rien, [...] remontent les ruisseaux à truites... », alors que cela est absolument impossible. Mais rares sont également les recherches qui tiennent compte précisément du milieu global : les cas étudiés portent plutôt sur des espèces emblématiques, abordent très peu l'aspect systémique et se limitent à un instant donné.

À la seconde étape, les impacts potentiels constituent une hypothèse de recherche qui permet de déterminer les analyses scientifiques nécessaires pour mettre en évidence des impacts réels, mesurés. Ces impacts potentiels doivent envisager, de manière exhaustive, toutes les externalités négatives. Malheureusement, la plupart des études s'arrêtent, à cette étape, sur «l'hypothèse du pire».

La mesure des impacts doit être étroitement corrélée à la fréquentation du point analysé pour pouvoir généraliser les résultats obtenus. Or, mesurer celle-ci suppose un travail lourd et onéreux. De plus, il est indispensable de relativiser les impacts par rapport à des entités écologiques pertinentes. Si opérer une telle relativisation relève d'une position écocentrée, cela renvoie aussi à des concepts écologiques tels que la connectivité et la fonctionnalité. Les études incluant ces deux aspects sont presque inexistantes dans les documents dépouillés.

\footnotetext{
${ }^{5}$ Les sources sont très diverses : livres, articles scientifiques, rapports d'études et de recherches, littérature grise...
} 
Leur validité est donc limitée pour les raisons exposées ci-dessus, mais d'autres éléments peuvent encore être évoqués.

Sauf impact massif et évident, les résultats scientifiquement prouvés, quand ils existent, restent toujours très en deçà des besoins de connaissances pour évaluer les changements éventuels dus à l'influence des sports de nature. Cette situation produit donc une incertitude, source de "controverse" (Callon et al., 2001). Or, les commanditaires attendent des préconisations des études engagées. Le scientifique se trouve alors dans l'obligation de produire une "expertise», une "connaissance raisonnable aussi objectivement fondée que possible» (Roqueplo, 1997, p. 44), et émet toujours des réserves sur celle-ci. Mais le commanditaire, en les « oubliant», peut faire évoluer le propos dans le sens qui lui convient.

On peut également relever des montées en généralité abusives qui concernent des résultats locaux, périodiques, partiels, transformés en affirmations générales, des transferts de résultats d'une zone, d'un écosystème ou d'une espèce particulière à d'autres éléments non analogues. Les exemples ne manquent pas et concernent aussi bien une minoration qu'une majoration de l'impact.

Autre aspect de la controverse, les auteurs des études émettent des préconisations parfois totalement déconnectées des résultats obtenus. Ainsi, Cernuska (1986), étudiant, pour le Conseil de l'Europe, les conséquences du ski d'été, conclut à un faible impact à l'exception de la trace de remontée où la végétation est endommagée, mais affirme (p. 95) : "Eu égard à son impact écologique et à son faible rôle économique, le ski sur herbe devrait être totalement interdit », alors que l'aspect économique n'a même pas été abordé.

\section{Les acteurs face à l'impact environnemental de leur activité}

L'impact environnemental des sports de nature est donc très difficile à caractériser d'un point de vue scientifique, ce qui contribue à exacerber les conflits entre les catégories d'acteurs. Pour ces derniers, la validité d'une étude dépend étroitement du commanditaire, si bien que l'ensemble des expertises fait l'objet d'une mise en question par les protagonistes qui ne savent plus très bien quels résultats sont valides et où commencent les interprétations.

Cette situation est encore confortée par deux phénomènes antagonistes. D'une part, les sportifs se considèrent comme respectueux de l'environnement ${ }^{6}$ tel qu'ils se le représentent : il existe un décalage important entre

\footnotetext{
${ }^{6}$ Comme le montre la réponse stéréotypée et systématique faite lorsque leur comportement est mis en cause, le seul souci environnemental commun à tous les sportifs étant de laisser les lieux propres.
}

l'impact de leur pratique tel qu'ils le perçoivent et tel qu'il peut être analysé par des scientifiques. Börner et Coffre (1999) mentionnent ainsi la mauvaise interprétation des parapentistes, qui croient parfois qu'un rapace joue avec eux, alors qu'il a un comportement agressif dénotant un dérangement. D'autre part, les naturalistes stigmatisent l'attitude des sportifs qui, dérangeant un oiseau ou un mammifère (Roche et d'Andurain, 1993 ; Buhot, 1999), ne cessent pas pour autant leur activité «coupable», confondant alors ignorance et «mauvaise volonté ». Cela peut biaiser sérieusement leur analyse de l'activité et donc leurs résultats.

Mais l'incertitude scientifique peut aussi être manipulée par les acteurs : elle représente une importante source de pouvoir, surtout dans des contextes conflictuels. Dans ce type de situation, l'impact environnemental est ainsi souvent confondu, volontairement ou non, avec le conflit d'usage. Cet amalgame conduit à radicaliser des oppositions entre groupes d'acteurs, car la mise en cause inadéquate est vécue comme une accusation injustifiée : les tentatives d'exclusion des milieux communs de pratique par l'un des protagonistes (Mounet, 1996) sont une situation courante dans les conflits d'usage (Ivy et al., 1992).

Différemment perçue par les acteurs concernés, confrontée à une connaissance imparfaite du double point de vue de l'écologie et des sports de nature, confondue souvent avec des conflits d'usage, l'évaluation de l'impact environnemental des sports de nature conduit à une incertitude scientifique et renvoie donc fondamentalement aux intérêts et aux valeurs de chacun des protagonistes en présence. C'est la raison pour laquelle l'instrument d'évaluation environnementale construit pour le PDESI de l'Ardèche renverse la perspective habituelle et propose une prise en compte d'enjeux environnementaux partagés par naturalistes et sportifs.

\section{L'instrument d'évaluation environnementale et le jeu des acteurs}

L'objectif assigné à l'instrument est de situer aisément un niveau d'enjeu environnemental sans réaliser une étude d'impact au coût cumulé rédhibitoire ${ }^{7}$ et qui ne pourrait déboucher sur des certitudes avérées. Il est fondé sur une hiérarchisation départementale spécifique de ces enjeux et concerne des espaces institutionnalisés comme lieux sportifs, bénéficiant de crédits publics pour leur équipement et leur fonctionnement et supportant des flux significatifs. Il a été adopté à l'unanimité par la CDESI, mais il est nécessaire de faire un retour critique sur sa conception et sur son utilisation.

\footnotetext{
7 En janvier 2004, l'inventaire en cours faisait état de 1050 espaces, sites et itinéraires ardéchois identifiés.
} 
Le premier aspect porte sur la gestion de l'incertitude scientifique par les protecteurs de la nature. Dans le passé, les sportifs ont souvent perçu comme infondées et imprévisibles les objections de ces derniers à leurs projets. Cet instrument présente l'avantage de réduire cette incertitude et la source de pouvoir qui lui est attachée, car la règle du jeu de l'évaluation est clairement annoncée. Mais plusieurs aspects viennent tempérer cette affirmation.

Le choix des entités à protéger a été le fait des seuls protecteurs de la nature, détenteurs des données indispensables pour avoir une vue globale des enjeux environnementaux. Les acteurs sportifs, assurés de la présence d'un médiateur, ont accepté l'expertise des protecteurs de la nature, car la plupart d'entre eux sont totalement ignorants de ces enjeux. Cette situation donne donc carte blanche à l'un des protagonistes sans que soit envisagée une régulation des pouvoirs publics, ce qui souligne fortement l'absence d'une expertise publique de l'environnement (Lascoumes, 1994) ${ }^{8}$. Mais une démocratie participative (Mermet, 2001) est-elle réellement possible sans un «égal accès » aux débats (Callon et al., 2001) pour tous les acteurs concernés?

C'est également le passage du scientifique au politique (Latour, 1991) qui est interrogé par cette situation, puisque les sportifs ont du mal à réfuter le pouvoir absolu de la science, qui a la capacité de transformer la nature ordinaire en nature patrimoniale : aucun n'a contesté la définition des enjeux choisis qui, pourtant, à catégorie de protection équivalente, sont évalués différemment dans d'autres départements.

L'instrument était fondé sur la concertation, mais il n'y a pas eu de réel travail de définition en commun des enjeux. Ni les analyses pour les caractériser, ni le suivi des situations pourtant explicitement définies comme requérant une gestion commune de l'incertitude scientifique, n'ont été abordés. Bien au contraire, et dès le départ, un certain nombre d'acteurs ont littéralement «naturalisé » l'instrument en l'assimilant à une formalisation du contexte d'action. Dès lors, le jeu sur les règles formelles est réapparu avec une tentative d'exploiter les zones d'incertitudes (Friedberg, 1993). La «mise en réseau » souhaitée (Callon, 1986; Latour, 1992) a donc au moins partiellement échoué, certains se repositionnant d'entrée en " coopération conflictuelle » : l'utopie du développement durable, si elle a le mérite de poser l'équilibre entre les acteurs comme un prérequis fondamental et de ne pas se contenter de traiter le seul aspect environnemen$\mathrm{tal}^{9}$, ne peut très certainement être entretenue que par

\footnotetext{
8 Il est à noter que le service environnement du conseil général n'a pas été associé à la démarche, mais seulement invité au moment de l'adoption de l'instrument; cela illustre la difficile articulation des politiques publiques, même au sein d'une seule collectivité territoriale.

${ }^{9}$ Les autres aspects, économiques et sociaux, ont fait l'objet d'un travail parallèle.
}

l'existence d'une médiation ininterrompue, gage d'une réelle concertation.

Enfin, cette analyse montre, sans surprise, la complémentarité des approches biologiques et sociales, les connaissances naturalistes de la protection de l'environnement devant être complétées par la nécessaire coordination des acteurs ainsi que par le difficile passage des résultats scientifiques à la négociation politique.

\section{Références}

Balland, P., Legrain, D., Duchamp, J., Laurain, C., Prats, M., 2003. La Contribution des départements à la politique de protection des espaces naturels. Rapport de l'Inspection générale de l'environnement, Ministère de l'Écologie et du Développement durable, Paris.

Börner, F., Coffre, H., 1999. Les Rapaces rupestres et les activités sportives de montagne. Rapport CORA Isère, Grenoble.

Bourdieu, P., 1979. La Distinction : critique sociale du jugement, Paris, Éditions de Minuit.

Buhot, D., 1999. Les enseignements de quelques expériences de gestion du vol libre en Vanoise. Communication au Cinquième forum des gestionnaires, organisé par Réserves naturelles de France, Espaces naturels de France, Fédération des PNR de France, Paris, 12 mars.

Callon, M., 1986. Éléments pour une sociologie de la traduction, Année sociologique, XXXVI, 86, 169-207.

Callon, M., Lascoumes, P., Barthe, Y., 2001. Agir dans un monde incertain : essai sur la démocratie technique, Paris, Le Seuil.

Cernuska, 1986. Les Répercussions écologiques de la construction et de l'exploitation des pistes de ski, Strasbourg, Conseil de l'Europe.

Deprest, F., 1997. Enquête sur le tourisme de masse : l'écologie face au territoire, Paris, Belin.

Friedberg, C., Cohen, M., Mathieu, N., 2000. Faut-il qu'un paysage soit ouvert ou fermé? L'exemple de la pelouse sèche du causse Méjan, Natures Sciences Sociétés, 8, 4, 26-42.

Friedberg, E., 1993. Le Pouvoir et la règle : dynamiques de l'action organisée, Paris, Le Seuil.

Ivy, M.I., Stewart, W.P., Lue, C.-C., 1992. Exploring the role of tolerance in recreational conflict, Journal of Leisure research, 24, 4, 348-360.

Jacob, G.R., Schreyer, R., 1980. Conflict in outdoor recreation: a theoretical perspective, Journal of Leisure Research, 12, 4, 368-380.

Larrère, C., 1997. Les Philosophies de l'environnement, Paris, PUF.

Larrère, C., et Larrère, R., 1997. Du bon usage de la nature : pour une philosophie de l'environnement, Paris, Aubier.

Lascoumes, P., 1994. L'Éco-pouvoir : environnements et politiques, Paris, La Découverte.

Latour, B., 1991. Nous n'avons jamais été modernes, Paris, La Découverte.

Latour, B. (Ed.), 1992. Ces réseaux que la raison ignore, Paris, L'Harmattan.

Lecomte, J., 2002. À la recherche de la nature, Le Courrier de l'environnement de l'INRA, 45, 17-22.

Lepart, J., Marty, P., Rousset, O., 2000. Les conceptions normatives du paysage. Le cas des Grands Causses, Natures Sciences Sociétés, 8, 4, 16-25. 
Mermet, L., 2001. L'institution patrimoniale du Haut Béarn : gestion intégrée de l'environnement ou réaction antienvironnementale?, Les Annales des Mines / Responsabilité $\mathcal{E}$ Environnement, 21, 9-21.

Mougenot, C., 2002. Prendre soin de la nature ordinaire, Paris, MSH/INRA.

Mounet, J.-P., 1996. Sports d'eau vive et pêche en rivière : un conflit asymétrique, STAPS, 40, 4-20.

Mounet, J.-P., 2000. Impact des activités sportives de nature sur le milieu humain, Cahiers Espaces, 67 (Tourisme durable).

Mounet, J.-P., Rocheblave, M., Nicollet, J.-P., 2000. L'impact des activités sportives de nature sur l'environnement naturel, Montagnes méditerranéennes, 11, 67-76.

Pociello, C., 1981. La force, la grâce et les réflexes, in Pociello, C., Sports et société : approche socioculturelle des pratiques, Paris, Vigot.
Roche, J., d'Andurain, P., 1993. Avifaune et sports d'eau vive dans les gorges du Haut Allier. Rapport d'étude, Ligue pour la protection des oiseaux, Clermont-Ferrand.

Roqueplo, P., 1997. Entre savoir et décision, l'expertise scientifique, Paris, INRA.

Stankey, G.H., Cole, D.N., Lucas, R.C., Peterson, M.E., Frissell, S.J., 1985. The limits of acceptable change (LAC) of wilderness planning. Forest Service Technical Report, Int-176, USDA, Ogden (Utah).

Winckel, C., 1999. La Fréquentation des milieux naturels : impacts $d u$ public et propositions. Mémoire de diplôme universitaire de $3^{\text {e }}$ cycle, Faculté libre des sciences et technologies, Lille.

To access this journal online: www.edpsciences.org 